\title{
CUIDADOS PALIATIVOS E MELHORIA DA QUALIDADE DE VIDA DOS PACIENTES ONCOLÓGICOS
}

\author{
Aline Gisela Souza Tavares*, Júlia Sousa Santos Nunes** \\ Autora correspondente: Aline Gisela Souza Tavares - alinegisela@hotmail.com \\ * Faculdade Independente do Nordeste em Vitória da Conquista - BA \\ ** Enfermeira, Graduada em Enfermagem, pós-graduada em Obstetrícia e Auditoria do Sistema de Saúde e mestre em \\ Saúde Coletiva - Gestão de Saúde pela Universidade Federal da Bahia
}

\section{Resumo}

O câncer é uma doença crônica degenerativa de evolução progressiva, caracterizado por um crescimento anormal e/ou acelerado das células que sofrem diversas mutações durante o processo de mitose e meiose. O processo visto a partir dos cuidados paliativos vê, contempla, analisa, descreve, destaca, interage, determina e orienta, cada paciente individualmente com seus problemas e suas limitações. O presente estudo tem como objetivo geral identificar se os pacientes oncológicos receberam algum tipo de cuidado paliativo para alívio dos sintomas associados ao câncer. Quanto aos objetivos específicos, este visa verificar junto aos pacientes a presença de sinais e sintomas associados a patologia; avaliar se os sinais e sintomas apresentados causam algum tipo de limitação física; identificar o tipo de intervenção realizada pelos profissionais de saúde na presença dos sintomas apresentados e avaliar o impacto dos cuidados paliativos na qualidade de vida do paciente. A metodologia utilizada na pesquisa teve uma abordagem qualitativa, de natureza descritiva com caráter exploratório, onde os sujeitos da pesquisa foram 20 pacientes que estavam em acompanhamento na casa do Amor em Vitória da Conquista - BA. Os dados obtidos através do questionário foram agrupados por categorias, depois de quantificados foram tabulados e lançados em planilha, representados em forma de gráficos e discutidos por categorias. Os resultados evidenciaram que $80 \%$ da população em estudo referiu ter conhecimento sobre a doença oncológica em tratamento e apenas $20 \%$ apresentaram dúvidas sobre a doença e o tratamento e estas foram solucionadas pelos profissionais, obtendo $100 \%$ para o esclarecimento. Observa-se que dos entrevistados $35 \%$ possui limitação física, $40 \%$ disseram sentir dor, vômitos e náuseas e todos informaram ter recebido prescrição médica para alívio dos sintomas. E ainda $40 \%$ participantes da pesquisa informaram obter falta de apetite e $35 \%$ paciente disseram ter ansiedade. Os cuidados paliativos não é uma tarefa fácil, mas requer atenção a pequenos detalhes como o conhecimento específico, cuidados de forma integral, apoio psicológico, orientação com esclarecimento de duvidas e apoio familiar.

Palavra Chave: Cuidados paliativos; Câncer; Qualidade de Vida 


\title{
HOSPICE AND IMPROVING THE QUALITY OF LIFE OF PATIENTS CANCER
}

\begin{abstract}
Cancer is a chronic progressive degenerative disease characterized by abnormal and / or accelerated growth of cells that undergo several changes during the process of mitosis and meiosis. It is the second leading cause of death worldwide, and despite scientific and technological have favored the development and improvement of the treatment of this pathology, showing a higher survival and healing in cancer patients as well as significant improvement in the level of quality of life improvements. This study's general objective is to identify the cancer patients received some form of palliative care for the relief of symptoms associated with cancer and aimed at verifying with patients for signs and symptoms associated with pathology; assess whether the signs and symptoms presented cause some kind of physical limitation; identify the type of intervention by health professionals in the presence of symptoms, and assess the impact of palliative care on quality of life of the patient. The methodology used in the research had a qualitative approach with descriptive exploratory nature, where the subjects were 20 patients who were followed at the home of Love in Vitória da Conquista, Bahia during this period. As results found in the present study there was a higher frequency of males (55\%) aged between 62 and 76 years, all literate. Therefore, the nursing process need further grounding, reflection on the objects and models, determination in the pursuit of improved tools for collecting.
\end{abstract}

Keyword: Hospice; Cancer; Quality of Life.

\section{INTRODUÇÃO}

O câncer é uma doença crônica degenerativa onde as células se dividem desordenadamente e de forma agressiva, formando tumores malignos e com potencial de desenvolver metástase em outros órgãos. As doenças oncológicas são consideradas problema de saúde pública por apresentarem elevada incidência, prevalência, mortalidade, gastos hospitalares e, acima de tudo, as consequências sobre a qualidade de vida do sujeito, bem como demandas de cuidado para os profissionais de saúde. É aí que entram os cuidados paliativos, para oferecer uma melhor qualidade de vida a estes pacientes. ${ }^{(1)}$

O câncer atualmente é a segunda maior causa de morte no mundo ocidental, principalmente nos países desenvolvidos e em desenvolvimento. Nos últimos anos vultosos investimentos em pesquisas por parte dos governos e de iniciativas privadas, além de um crescente número de trabalhos científicos publicados na área de oncologia, confirmam a importância e preocupação atribuída à esta doença. ${ }^{(2)}$

A história da cancerologia é conhecida desde a Grécia Antiga, com 2000 anos de hipóteses, estudos e suportes tecnológicos para o tratamento e diagnóstico. $\mathrm{Na}$ atualidade o processo de oncologia tornou-se um problema de saúde pública para o Brasil, isto se dá pelos altos índices de morbimortalidade em casos e prevalência. ${ }^{(3)}$

No ano de 2007, o Instituto Nacional do Câncer (INCA), do Ministério da Saúde, analisando as taxas de mortalidade no Brasil, constatou que o câncer está incluído entre as principais causas de morte, só perdendo para as doenças do apareIho circulatório, ao lado de causas externas, doen- 
ças do aparelho respiratório, afecções do período perinatal, doenças infecciosas e parasitárias. Esta doença é a segunda causa de morte no país, sendo responsável por 10,86\% dos óbitos ocorridos em 1994; sendo que $53,81 \%$ entre os homens e $46,05 \%$ entre as mulheres. ${ }^{(4)}$

A organização Mundial de Saúde (OMS) estima que onze milhões de pessoas sejam diagnosticados com câncer por ano, e que a doença representa 12,5\% das mortes no mundo. Segundo a mesma mais de 16 milhões de casos serão verificados até 2020.(5)

Os Cuidados Paliativos originaram-se no movimento hospices. Em 1840, na França, os hospices serviam de abrigos para peregrinos. O termo Palliare significa proteger, amparar, cobrir, abrigar. Cuidados paliativos tem como objetivo principal a qualidade de vida e não somente o tempo que ainda se tem de vida, fornecendo alívio da dor, dos sintomas estressantes e ansiedade, reafirmar a vida e a morte como processo natural, não apressar ou adiar a morte. Objetiva também oferecer um sistema de apoio à família para lidar com a doença do paciente, ajudar o paciente a ter uma vida ativa e não ficar somente esperando a morte, além de aconselhamento e suporte ao luto. ${ }^{(6)}$

O maior desafio dos cuidados paliativos é agir simultaneamente com os cuidados curativos, pois muitos profissionais de saúde desconhecem sua importância e a maioria das instituições não tem isso bem definido. O interesse pelo tema surgiu a partir de uma vivência com uma pessoa idosa diagnosticada com câncer de esôfago que por causa da idade, não foi possível fazer o tratamento completo, optou-se por aplicar os cuidados paliativos afim de fornecer um final de vida digno, sem dores e ao lado das pessoas da família. ${ }^{(7)}$

Nesta perspectiva, o presente estudo tem como objetivo geral identificar se os pacientes oncológicos receberam algum tipo de cuidado paliativo para alívio dos sintomas associados ao câncer e como objetivos específicos verificar junto aos pacientes a presença de sinais e sintomas associados a patologia; avaliar se os sinais e sintomas apresentados causam algum tipo de limitação física; identificar o tipo de intervenção realizada pelos profissionais de saúde na presença dos sintomas apresentados e avaliar o impacto dos cuidados paliativos na qualidade de vida do paciente.

A finalidade desta pesquisa é cooperar com a reflexão e a abrangência sobre descrição e todos os artifícios que decompõe esta patologia, tendo em vista o mais importante que é a observação metodológica que foi identificada e realizada para fins de conhecimento de dados para o cuidado paliativo a população portadora de câncer.

\section{MÉTODOS}

A metodologia utilizada na pesquisa teve uma abordagem qualitativa, de natureza descritiva. Além de ser direcionada ao longo do seu desenvolvimento, a pesquisa qualitativa não busca enumerar ou medir eventos e, geralmente, não emprega instrumentos estatísticos para análise dos dados; tem amplo foco de interesse e parte de uma perspectiva diferenciada da adotada pelos métodos quantitativos. Os dados descritivos são obtidos mediante contato direto e interativo do pesquisador com o objeto de estudo.(8)

Dado o seu caráter exploratório, as pesquisas qualitativas não pretendem generalizar as suas informações, não havendo, portanto, preocupação em projetar os seus resultados para a população. Abordam-se, em geral, pequenos grupos de entrevistados. ${ }^{(8)}$

A amostra é probabilística por conveniência e delimita-se como sujeito da pesquisa os pacientes em acompanhamento na Casa do Amor na cidade de Vitória da Conquista que estavam presentes na instituição no período da pesquisa. Foi adotado o seguinte critério de inclusão: pacientes portadores de patologias oncológicas que estiveram presentes na instituição no momento da coleta de dados e que aceitaram participar voluntariamente da pesquisa e assinarem o Termo de Consentimento Livre e Esclarecido. Como critérios de exclusão tem-se: àqueles que se negaram a participar, aqueles que não foram encontrados na instituição e os que estiveram totalmente ou parcialmente incapacitados fisiologicamente ou psicologicamente. 
A pesquisa foi realizada em julho de 2014 , com aplicação de questionário semiestruturado com questões relacionadas ao estudo, aplicado pelo pesquisador com o objetivo de avaliar a aplicação dos cuidados paliativos em pacientes portadores de doença oncológica e a repercussão na qualidade de vida. Participaram da pesquisa 20 pacientes que estavam em acompanhamento na casa do Amor neste período.

Os dados obtidos através das entrevistas foram transcritos e agrupados por categorias. Os dados quantificados foram tabulados e, representados em forma de tabela e discutidos por categorias de forma descritiva.

Esta pesquisa foi aprovada pelo Comitê de Ética em Pesquisa da Faculdade Independente do Nordeste (CEP/FAINOR). Número do Parecer: 684.843 de $11 / 06 / 2014$.

\section{RESULTADOS E DISCUSSÃO}

Constatou-se no presente estudo que houve uma maior frequência de indivíduos do sexo masculi- no $(55,0 \%)$ com idade entre 62 e 76 anos, todos alfabetizados.

De acordo com o INCA, baseado na Estimativa 2014, há uma prevalência das doenças oncológicas na população acima dos 60 anos, com maior incidência e mortalidade na população masculina. Sem considerar os casos de câncer de pele não melanoma, estimam-se 395 mil casos novos de câncer, 204 mil para o sexo masculino e 190 mil para sexo feminino, sendo que em homens, os tipos mais incidentes serão os cânceres de próstata, pulmão, cólon e reto, estômago e cavidade oral; e, nas mulheres, os de mama, cólon e reto, colo do útero, pulmão e glândula tireoide. Dentre as causas citadas predomina o envelhecimento da população. (9)

Nota-se que os dados encontrados no estudo estão em acordo com a referência acima citada, indicando uma necessidade de maior atenção da política de Atenção a Saúde do Homem e a política de Atenção à Saúde do Idoso priorizando o controle e a prevenção.

Em relação ao tipo de câncer houve uma maior distribuição de CA de Próstata $(35,0 \%)$ e de CA de mama (30,0\%), conforme Tabela 1.

Tabela 1 - Distribuição percentual em relação aos tipos de CA

\begin{tabular}{lcc}
\hline & $\mathbf{N}$ & $\%$ \\
\hline CA DE PROSTATA & 7 & 35,0 \\
CA DE MAMA & 6 & 30,0 \\
CA DE ESÔFAGO & 2 & 10,0 \\
CA DE INTESTINO & 1 & 5,0 \\
CA DE PELE MELANOMA & 1 & 5,0 \\
CA DE ÚTERO & 1 & 5,0 \\
CA DE ESTÔMAGO & 1 & 5,0 \\
CA DE LARINGE & 1 & 5,0 \\
\hline TOTAL & 20 & 100 \\
\hline
\end{tabular}

Fonte: dados da pesquisa realizada na casa do Amor, Vitória da Conquista/BA, 2014.

Neste contexto prevalece em maior caso o câncer na próstata, dados mostram que em 2010 foram registradas 12.778 mortes provenientes do câncer de próstata, sem considerar o número de diagnóstico e as pessoas em que não tiveram no obituário, o registro da doença, tendo sido estima- 
do para 2012 é de 60.180 casos diagnosticados, o que corresponde a um risco estimado de 62 casos novos a cada 100 mil homens. ${ }^{(9)}$

O câncer de próstata é o mais comum entre os homens com idade igual ou superior a 65 anos, por isso é considerado um câncer da terceira idade. $O$ aumento observado nas taxas de incidência no Brasil pode ser parcialmente justificado pela evolução dos métodos diagnósticos (exames), pela melhoria na qualidade dos sistemas de informação do país e pelo aumento na expectativa de vida. Já o câncer de mama é o tipo de câncer que mais acomete as mulheres em todo o mundo, e representa a maior causa de morte por câncer nas mulheres em todo o mundo, com cerca de 520 mil mortes estimadas para o ano de 2012.(10)

Apesar de ser considerado um câncer de relativamente bom prognóstico, se diagnosticado e tratado oportunamente, as taxas de mortalidade por câncer de mama continuam elevadas no Brasil, muito provavelmente porque a doença ainda é diagnosticada em estágios avançados.

Em 2006 foram registrados no total de casos novos o câncer de pele não-melanoma ( 116 mil casos novos) sendo o mais incidente na população brasileira, seguido pelos tumores de mama feminina (49 mil), próstata (47 mil), pulmão (27 mil), cólon e reto ( $25 \mathrm{mil})$, estômago $(23 \mathrm{mil})$ e colo do útero (19 mil). Os tumores mais incidentes no sexo masculino, excluindo-se o câncer de pele não-melanoma, são os de próstata, pulmão, estômago e cólon e reto. No sexo feminino, destacam-se os tumores de mama, colo do útero, colón e reto e pulmão. ${ }^{(9)}$

Esses dados puderam ser confirmados durante a pesquisa uma vez que a maioria dos homens que responderam o questionário apresenta câncer de próstata e apresentam idade igual ou superior a 65 anos, já entre as mulheres o câncer de mama prevalece, sendo encontrado também casos das outras doenças oncológicas de maior prevalência.

Vale ressaltar que ações de prevenção e controle do câncer estão diretamente ligadas a desafios educacionais, culturais e científicos, incremento em políticas de saúde pública, emprego metodológico e racional dos recursos disponíveis em nossa época. Compreender e controlar as doenças oncológicas requer muito mais que conhecimentos científicos. Exige-se aporte de experiências e estratégias administrativas que vão muito além do conhecimento dos complexos mecanismos de regulação molecular intracelular. Também se exige gestão competente, avaliação e planejamento destas estratégias visando melhor controle e execução dessas ações preventivas. ${ }^{(7)}$

A cerca do conhecimento sobre a doença oncológica em tratamento, $80,0 \%$ da população em estudo referiu ter conhecimento, e apenas $20,0 \%$ dos entrevistados apresentaram dúvidas sobre a doença e o tratamento e estas foram solucionadas pelos profissionais, assim em relação às dúvidas sobre a doença $85,0 \%$ não apresentam dúvidas, obtendo $100 \%$ para o esclarecimento como mostra a Tabela 2.

Tabela 2 - Distribuição percentual em relação ao conhecimento da doença, a dúvidas e Esclarecimentos dados pelo profissional de saúde

\begin{tabular}{lcc}
\hline & $\mathbf{N}$ & $\mathbf{\%}$ \\
\hline CONHECEM A DOENÇA & 16 & 80,0 \\
DESCONHECEM A DOENÇA & 4 & 20,0 \\
DÚVIDAS SOBRE A DOENÇA & 3 & 15,0 \\
NÃO TEM DÚVIDAS & 17 & 85,0 \\
ESCLARECIMENTO DE DÚVIDAS & 3 & 100 \\
NÃO SE APLICA & 17 & - \\
\hline TOTAL & 20 & 100
\end{tabular}

Fonte: Dados da pesquisa realizada na casa do Amor, Vitória da Conquista/BA, 2014. 
Atualmente no Brasil existem políticas voltadas à prestação de assistência adequada aos pacientes portadores de neoplasia, já que o câncer é considerado um problema de Saúde Pública. A Política Nacional de Atenção Oncológica, agrupada pela Portaria $n^{\circ} 2.048$, de 3 de setembro de 2009 do Ministério da Saúde, determina, para o país, efetivo controle do câncer, e considera vários artefatos, desde as ações voltadas à prevenção até a assistência de alta complexidade, integradas em redes de atenção básica de saúde, com o objetivo de minimizar os danos causados à população e reduzir a incidência de mortalidade por câncer. Assim os profissionais de saúde que lidam diretamente com esses pacientes portadores de neoplasias devem conhecer todo ciclo patológico no intuito de oferecer um melhor atendimento e estabelecer novos hábitos de vida saudável para obter uma meIhoria na qualidade de vida, requesito fundamental para que possamos mudar esta realidade em nosso país. ${ }^{(11)}$

A formação dos profissionais de enfermagem é orientada para uma visão generalista tendo em vista os aspectos sociais do nosso país. Entretanto devido à complexidade das necessidades do paciente oncológico, os profissionais de enfermagem com formação específica faz-se imprescindível no planejamento assistencial para suprir as necessidades farmacológicas, terapêuticas fisiológicas e psicossociais do paciente oncológico, destaca-se também a importância dos profissionais que contribuem cientificamente para o avanço de técnicas da assistência a estes pacientes. ${ }^{(9)}$

É muito importante que o paciente conheça seu diagnóstico para poder conversar com o médico sobre o melhor tratamento para sua doença, porém é sabido que o tratamento do câncer não é fácil e nem rápido e que a quimioterapia e radioterapia além de apresentar vários efeitos colaterais como fadiga, dispneia, queda de cabelo também atinge a autoimagem do paciente, por isso tanto os familiares como o paciente devem receber esclarecimentos sobre os riscos benefícios dos tra- tamentos oferecidos para conseguir a adesão do paciente.

O processo dos cuidados paliativos hoje avaliado como um acontecimento de origem complexa e multifatorial, envolve aspectos neurológicos orgânicos e psicossociais. As terapêuticas neurofisiológica e neurofarmacológico são consideradas eficazes, mas a abordagem multidisciplinar, a psico-oncologia, tem importância ímpar para a qualidade de vida do paciente portador de câncer, as sim faz importante os esclarecimentos de dúvidas, para assim manter o paciente esclarecido sobre todo o processo do tratamento, talvez o mesmo pode se sentir mais seguro e para a estar realizando de maneira adequada. ${ }^{(10)}$

Assistir um paciente em oncologia harmonicamente é proporcionar acréscimo da expectativa de vida desse indivíduo qualitativamente, e não meramente a cura patológica. Mesmo operando sob o atual modelo biomédico, o cuidado de enfermagem, por sua vez, tem, em sua profundeza, assistir ao ser humano em seu contexto mais íntimo, observando a relação bio-socio-cultural. Portanto, a assistência de enfermagem no coloquial do cuidar, direciona-se para o ensino do autocuidado, com o objetivo de resguardar a autonomia e autoestima do paciente, agregando reconhecimento e valorização ao profissional, ao estabelecer esta relação positiva e empática entre quem cuida e quem é cuidado. $(4)$

Observa-se na Tabela 4, que dos entrevistados 7 (35,0\%) possui limitação física e $13(65,0 \%)$ não possuem, $8(40,0 \%)$ disseram sentir dor, vômitos e náuseas e todos informaram ter recebido prescrição médica para alívio dos sintomas, que 8 (40,0\%) participantes da pesquisa informaram obter falta de apetite e $12(60,0 \%)$ informação não apresentar inapetência e por fim 7 (35,0\%) paciente disseram ter ansiedade, sendo que o restante que representa $13(65,0 \%)$ não possui ansiedade para o quadro. 
Tabela 4 - Distribuição percentual em relação a limitação física, dor, vômitos/náuseas, falta de apetite e ansiedade

\begin{tabular}{lcc}
\hline & $\mathbf{N}$ & $\%$ \\
\hline LIMITAÇÃO FISICA & 7 & 35,0 \\
Sim & 13 & 65,0 \\
Não & & \\
DOR & 8 & 40,0 \\
Sim & 12 & 60,0 \\
Não & & \\
VÔMITO/NAUSEAS & 8 & 40,0 \\
Sim & 12 & 60,0 \\
Não & & \\
FALTA DE APETITE & 8 & 40,0 \\
Sim & 12 & 60,0 \\
Não & & 35,0 \\
ANSIEDADE & 7 & 65,0 \\
Sim & 13 & 100 \\
Não & 20 &
\end{tabular}

Fonte: dados da pesquisa realizada na casa do Amor, Vitória da Conquista/BA, 2014.

Neste contexto, observa-se um maior predomínio em relação à ausência de dor, inapetência e ansiedade, havendo uma porcentagem mínima de algias e limitação física. Além disso, $35 \%$ relatam ansiedade e referem não receber assistência na casa diminuir esse sintoma.

Em termo de morfologia, o parênquima tumoral ostenta um grau variado de células. As dos tumores de forma benignos, que são similares e reportam o aspecto das células do tecido que lhes deu ascendência, são denominadas bem diferenciadas. As células dos tumores malignos submergiram estas características, tendo graus variados de diferenciação, portanto, há diversos aspectos sintomatológicos como vômitos, algias, ansiedade, limitação ao realizar atividades, ate mesmo depressão. ${ }^{(12)}$

$\mathrm{Na}$ relação com as alterações fisiológicas cancerosas, o enfermeiro também expressa suas dificuldades em lidar com o sofrimento causado pela doença e suas dores onde surgem os diversos comportamentos da mudança de melhora de assistência por partes dos profissionais o enfermeiro deve estar apto para dar contribuição aos pacientes e suas respectivas famílias durante possíveis crises físicas, emocionais, sociais, culturais e espirituais. A abrangência dos objetivos almejados engloba oferecer um apoio realista aos clientes submetidos ao tratamento, usando padrões auxiliares com o processo de enfermagem agregando qualidade na assistência através do conhecimento cientifico adquirido. ${ }^{(13)}$

Daí surge o termo denominado de empatia que quer dizer uma resposta afetiva verídica a outras pessoas. Portanto cada profissional de enfermagem tem que se colocar no lugar do outro para prestar sua assistência de forma humanizada, pois todos tem direito a qualidade do atendimento, qualquer paciente seja ele oncológico ou não tem que haver uma assistência de forma correta. Deve haver prestação na assistência de forma holística, onde se lida com a realidade incluindo o processo de empatia. ${ }^{(11)}$

Baseado na discussão acima, verificou-se que $100 \%$ dos entrevistados recebem apoio religioso, dentro do próprio ambiente da Casa do Amor, o que favorecer ainda a recuperação ao paciente portador de neoplasias, bem como receberam cuidados prestados através da assistência de enfermagem e foram 
medicados para os sinais e sintomas apresentados decorrentes da patologia.

\section{CONSIDERAÇÕES FINAIS}

Ao findar o presente estudo percebe-se que os objetivos foram atingidos com êxito, identificou-se que os pacientes oncológicos em sua totalidade receberam algum tipo de cuidado paliativo para alívio dos sintomas associados ao câncer, como: dor, náuseas, vômitos, falta de apetite e ansiedade. Constatou-se que boa parte dos pacientes apresentou algum tipo de limitação decorrente da patologia e que todos foram atendidos em suas necessidades proporcionando uma melhor qualidade de vida a estes.

Diante da perspectiva de que uma grande parte dos sujeitos da pesquisa conhece a doença, e que possuem respostas para suas dúvidas em relação à mesma, obtendo os devidos esclarecimentos sobre o processo patológico, identifica-se uma assistência prestada voltada para a prática da enfermagem ao paciente oncológico é um processo complexo, uma vez que a capacidade de trabalhar com o ser humano em suas experiências de máxima intensidade não centra-se unicamente no bem-estar da pessoa, mas em seu existir mais pleno, ajudando o ser humano em um momento singular da sua vida. Com a análise das bibliografias em relação ao tema proposto foi possível criar numa visão do conhecimento da enfermagem em relação aos cuidados com os pacientes portadores de câncer.

O foco a respeito da assistência do no ato do cuidar, possui uma ciência visando a pratica onde é imprescindível o trabalho cotidiano dos profissionais enfermeiros e dos técnicos de enfermagem. $O$ cuidado a pessoa humana é uma colocação exercida no sentido de aumentar um processo de prática dentro dos princípios de valores éticos morais, fazendo parte de todos os grupos humanos, assim, o lidar com este ser vem tornando certo processo humanístico para uma estabilidade aos usuários.
Neste contexto diante da avaliação aos cuidados paliativos a educação do profissional que atua na enfermagem oncológica deve destacar a padronização de condutas, permitindo uma alteração dessas atitudes com o estudo dos casos e a experiência cientificamente adquirida. A enfermagem deve ter papel ativo no combate ao câncer e principalmente no que concerne a educação, orientação educacional e assistencial ao paciente e seus familiares.

Os profissionais da área da saúde, em destaque o enfermeiro, pela proximidade e interatividade inerente, deve estar informado de que a sua assistência é de essencial importância para estes pacientes, essa consciência deve permitir o usuário uma recuperação adequada estável. Os cuidados paliativos não é uma tarefa fácil, mas requer apenas atenção a pequenos detalhes, conhecimento específico para cada atendimento, visto que o paciente necessita de cuidados de forma integral, apoio psicológico e familiar. Todos os seres humanos e em particular o portador de doença oncológica tem direito a qualidade de vida e a prestação da assistência a saúde.

A presente pesquisa poderá servir de subsídio para que os profissionais de saúde, especialmente o enfermeiro procure trabalhar de forma mais enfática os cuidados paliativos voltados para o paciente oncológico, possibilitando a estes uma melhor qualidade de vida.

\section{REFERÊNCIAS}

1. Brasil. Ministério da Saúde. Sistema de Informações Ambulatoriais. Manual de Bases Técnicas em Oncologia. Brasília, 2006. Disponível em: http://www.ensp.fiocruz.br/portalensp/judicializacao/pdfs/305.pdf.

2. Oncoguia. O que é Câncer. 2011. Disponível em: http://www.oncoguia.com.br/site/interna. php?cat $=2 \&$ id $=474 \&$ menu $=2$

3. Rodrigues JSM, Ferreira NMLA. Caracterização do perfil epidemiológico do câncer em uma cidade do interior paulista: conhecer para intervir. Rev. bras. cancerol. 2010;56(4);431-441. Disponível em: http://www.inca.gov.br/rbc/n_56/ vO4/pdf/O5_artigo_caracterizacao_perfil_ 
epidemiologico_cancer_cidade_interior_paulista_ conhecer_para_intervir.pdf.

4. Menezes MFB et al. Câncer, pobreza e desenvolvimento humano: desafios para a assistência de enfermagem em oncologia. Rev. latinoam enferm. 2007;15(1):1-6. Disponível em: http://www.scielo.br/pdf/rlae/v15nspe/pt_1O.pdf.

5. Brasil. Ministério da Saúde. Instituto nacional de Câncer José Alencar Gomes da Silva. Nomenclatura brasileira para laudos cervicais e condutas preconizadas: recomendações para profissionais de saúde. Rio de Janeiro; 2006. Disponível em: http://bvsms.saude.gov.br/bvs/ publicacoes/Nomenclaturas_2_1705.pdf

6. Silva LMH, Zago MMF. O cuidado do paciente oncológico com dor crônica na ótica do enfermeiro. Rev. latinoam enferm. 2001;9(4):4449. Disponível em: http://www.scielo.br/pdf/rlae/ v9n4/11482.pdf

7. Brasil. Ministério da Saúde. Secretaria de Atenção à Saúde. Manual de bases técnicas: oncologia. 13a ed. Brasília; 2O11. Disponível em: http://wwwl.inca.gov.br/inca/Arquivos/manual_ oncologia_13edicao_agosto_2O11.pdf

8. Neves JL. Pesquisa qualitativa: características, usos e possibilidades. Caderno de Pesquisa em Administração. 1996;1(3):2-5.
9. Brasil. Ministério da Saúde. Instituto nacional de Câncer José Alencar Gomes da Silva. INCA lança estimativas de câncer 2012 com sete novas localizações de tumores. Rio de Janeiro; 2012. Disponível em: <http://www2.inca.gov. $\mathrm{br} / \mathrm{wps} / \mathrm{wcm} / \mathrm{connect/agencianoticias/site/}$ home/noticias/2011/inca_lanca_estimativas_de_ cancer_2O12_com_sete_novas_localizacoes_de_ tumores>.

10. Brasil. Ministério da Saúde. Instituto nacional de Câncer José Alencar Gomes da Silva. Fatores de risco: normas e manuais técnicos. Rio de Janeiro; 2010.

11. Araujo CRG, Rosas AMMTF. O papel da equipe de enfermagem no setor de radioterapia: uma contribuição para a equipe multidisciplinar. Rev. bras. cancerol. 2007;54(3):231-237. Disponível em: http://www.inca.gov.br/rbc/n_54/vO3/pdf/ artigo_4_pag_23la237.pdf.

12. Smeltzer SC, Bare BG. Brunner \& Suddarth: tratado de enfermagem médico-cirúrgica. $9^{\mathrm{a}} \mathrm{ed}$. Rio de Janeiro: Guanabara Koogan; 2002.

13. Brasil. Ministério da Saúde. Portaria n $2.048,3$ de Setembro de 2009. Aprova o Regulamento do Sistema Único de Saúde (SUS). Brasília; 2009. Disponível em: http://portal.saude.gov.br/ portal/arquivos/pdf/regulamento_sus_240909. pdf 